\title{
Two-Dimensional DOA Estimation in Compressed Sensing with Compressive-Reduced Dimension- $l_{p}$-MUSIC
}

\author{
Weijian Si, Xinggen Qu, Lutao Liu, and Zhiyu Qu \\ Department of Information and Communication Engineering, Harbin Engineering University, Harbin 150001, China
}

Correspondence should be addressed to Lutao Liu; liulutao@msn.com

Received 2 February 2015; Revised 17 May 2015; Accepted 28 May 2015

Academic Editor: Ananda S. Mohan

Copyright (C) 2015 Weijian Si et al. This is an open access article distributed under the Creative Commons Attribution License, which permits unrestricted use, distribution, and reproduction in any medium, provided the original work is properly cited.

\begin{abstract}
This paper presents a novel two-dimensional (2D) direction of arrival (DOA) estimation method in compressed sensing (CS) to remove the estimation failure problem and achieve superior performance. The proposed method separates the steering vector into two parts to construct two corresponding noise subspaces by introducing electric angles. Then, electric angles are estimated based on the constructed noise subspaces. In order to estimate the azimuth and elevation angles in terms of estimates of electric angles, arc-tangent operations are exploited. The arc-tangent is a one-to-one function and allows the value of the argument to be larger than unity so that the proposed method never fails. The proposed method can avoid pair matching to reduce the computational complexity and extend the number of snapshots to improve performance. Simulation results show that the proposed method can avoid estimation failure occurrence and has superior performance as compared to existing methods.
\end{abstract}

\section{Introduction}

Direction of arrival (DOA) estimation has been a topic of great interest in many fields such as radar, mobile communication systems, and medical imaging $[1,2]$. Many conventional DOA estimation methods have been developed in the last years. Among them, multiple signal classification (MUSIC) [3] and estimation of signal parameter via rotational invariance technique (ESPRIT) [4] are regarded as the most popular methods that have high resolution for DOA estimation. However, the performance of these methods can severely degrade when signal to noise ratio (SNR) is low, number of snapshots is small, or sources are coherent.

To overcome the aforesaid drawbacks, DOA estimation involving compressed sensing (CS) $[5,6]$ is investigated due to the development of methods based on CS. The CSbased estimation methods enforce sparsity on the spatial spectrum and perform source localization in an overcomplete dictionary. Malioutov et al. [7] propose the $l_{1}$-SVD method for DOA estimation, which casts DOA estimation problem as a sparse recovery problem. Stoica et al. [8] present a sparse iterative covariance-based estimation (SPICE) method by exploiting the covariance matching criterion. In [9], an alternative strategy called joint $l_{0}$ approximation method is proposed to resolve closed spaced and high coherent sources even if the number of sources is unknown.

Although one-dimensional (1D) DOA estimation has attracted tremendous interest, two-dimensional (2D) DOA estimation is of more practical importance. Since L-shaped array configuration has higher accuracy than other array configurations [10] such as the parallel uniform linear array (ULA) configuration [11], the rectangular array configuration [12], and the circular array configuration [13], most existing 2D DOA estimation methods are proposed based on the Lshaped array. Tayem and Kwon [14] propose the propagator method (PM) with one or two L-shaped array configurations to remove nonnegligible drawbacks of PM with parallel shape array configuration, but PM with one L-shaped array configuration still has estimation failure problem; that is, estimation method cannot perform estimation correctly in the entire ranges of the azimuth and elevation angles. Based on the L-shaped array configuration, Liang and Liu [15] propose a novel joint azimuth and elevation angles estimation method, which avoids pair matching. It has been proven that conventional 2D-MUSIC [16] method can provide a precise $2 \mathrm{D}$ estimation, but the requirement of $2 \mathrm{D}$ search needs high 
computation complexity. To reduce the computational load, a sparse L-shaped array configuration [17] is used, where the azimuth and elevation angles are estimated by the shiftinvariance property of ULA and modified total least squares (MTLS) techniques, respectively. In [18], Wang et al. propose $2 \mathrm{D}-l_{1}$-SVD and enhanced $2 \mathrm{D}-l_{1}$-SVD methods, which have several advantages over conventional methods.

In this paper, a novel compressive-reduced dimension$l_{p}$-MUSIC method called CS-RD- $l_{p}$-MUSIC, which requires no pair matching, is proposed for $2 \mathrm{D}$ DOA estimation in CS. The key idea of the proposed method is that the steering vector is separated into two parts to construct two corresponding noise subspaces by introducing electric angles. Then, based on the constructed noise subspaces, electric angles are estimated by the proposed method, where CSMUSIC is employed to estimate one electric angle and RD- $l_{p}$-MUSIC is adopted for other electric angles. What is more, CS-MUSIC can improve performance significantly even if covariance matrix tends to lose rank and $\mathrm{RD}-l_{p^{-}}$ MUSIC can reduce computational complexity by reduced dimension (RD). Our objective in this paper is to estimate the azimuth and elevation angles based on estimates of electric angles by arc-tangent operations. The arc-tangent is a oneto-one function and allows the value of its argument to be larger than unity in order that the proposed method never fails. We show that the proposed method can remove the estimation failure problem and achieve good performance due to the application of CS and extension of the number of snapshots. In addition, the proposed array configuration can further remove the estimation failure problem without loss of performance of DOA estimation. Simulation results illustrate the superior performance of the proposed method with comparisons to existing methods.

Notations used in this paper are given as follows. Lowercase boldface italic letters are served for vectors and uppercase boldface italic letters are served for matrices. $(\cdot)^{*}$, $(\cdot)^{T}$, and $(\cdot)^{H}$ denote the complex conjugate, transpose, and conjugate transpose, respectively. $(\cdot)^{-1}$ denotes the inversion of square matrix or pseudoinversion of nonsquare matrix. $\|\cdot\|_{2}$ and $\|\cdot\|_{p}$ denote the Euclidean norm for vectors and $l_{p^{-}}$ norm for matrices, respectively. $\mathbf{A}_{i}, \mathbf{A}^{i}$, and $A_{i, j}$ denote the $i$ th column, $i$ th row, and $(i, j)$ th entry of matrix $\mathbf{A}$, respectively. $\operatorname{diag}(\mathbf{A})$ is a diagonal matrix with the diagonal elements of matrix $\mathbf{A}$ and $\operatorname{diag}(\mathbf{a})$ is a diagonal matrix with a being its diagonal elements. $\mathbf{A}_{(K)}=\left[\mathbf{E}_{11}^{(K)}, \mathbf{E}_{22}^{(K)}, \ldots, \mathbf{E}_{K K}^{(K)}\right]^{T}$ is a $K^{2} \times K$ matrix, where $\mathbf{E}_{i i}^{(K)}, i=1,2, \ldots, K$, is a $K \times K$ matrix with one in the $(i, i)$ th entry and zeros elsewhere. $\mathbf{e}_{K}(i)$ is a $K \times 1$ vector with one in the $i$ th element and zeros elsewhere. $\mathbf{I}_{K \times K}$ is a $K \times K$ identity matrix. $\mathbf{0}_{K \times K}$ is a $K \times K$ matrix of all zeros. $\otimes$ and $\odot$ denote the Kronecker product and Hadamard product, respectively. unvec $(\cdot)$ denotes the matrix form of a vector. $\operatorname{Re}\{\cdot\}$ and $\operatorname{Im}\{\cdot\}$ denote the real and imaginary parts of a complex variable, respectively.

The remainder of the paper is organized as follows. In Section 2, we formulate the 2D DOA estimation problem, in which the steering vector is separated into two parts. The proposed method is described in detail in Section 3.

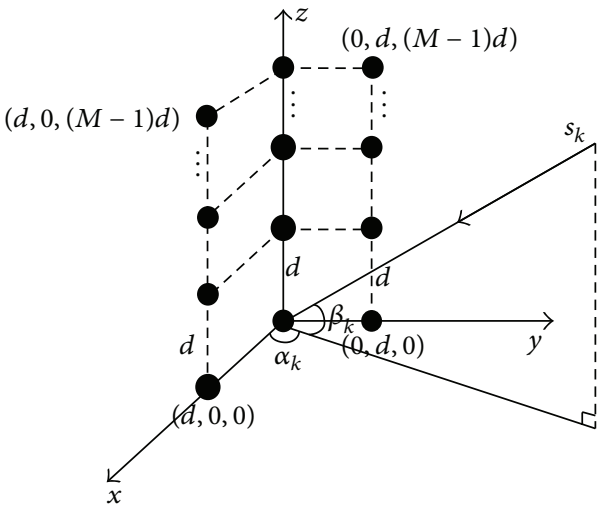

FIGURE 1: Proposed array configuration for 2D DOA estimation.

Section 4 shows the performance of the proposed method and Section 5 concludes the paper.

\section{Problem Formulation}

The proposed array configuration that consists of three uniform linear arrays (ULAs) of $M$ sensors with intersensor spacing $d$ is shown in Figure 1. One ULA lies in the $x-z$ plane, another lies in the $y-z$ plane, and the last one lies on the $z$-axis. The origin of the array configuration is set as the referencing sensor. Consider the array configuration impinged on $K$ narrowband far-field sources, $s_{k}(t), k=$ $1,2, \ldots, K$, where the $k$ th source has the azimuth angle $\alpha_{k}$ and elevation angle $\beta_{k}$ shown in Figure 1.

The coordinate of the $\left(i_{x}, i_{y}, i_{z}\right)$ th sensor is $\left(i_{x} d, i_{y} d, i_{z} d\right)$ so that the received source on the $\left(i_{x}, i_{y}, i_{z}\right)$ th sensor at the $t$ th snapshot can be given by

$$
\begin{aligned}
& x_{i_{x}, i_{y}, i_{z}}(t) \\
& =\sum_{k=1}^{K} s_{k}(t) e^{-j 2 \pi d\left(\cos \beta_{k} \cos \alpha_{k} i_{x}+\cos \beta_{k} \sin \alpha_{k} i_{y}+\sin \beta_{k} i_{z}\right) / \lambda} \\
& \quad+n_{i_{x}, i_{y}, i_{z}}(t) \\
& =\sum_{k=1}^{K} s_{k}(t) e^{j \theta_{k} i_{x}} e^{j \varphi_{k} i_{y}} e^{j \phi_{k} i_{z}}+n_{i_{x}, i_{y}, i_{z}}(t)
\end{aligned}
$$

where $n_{i_{x}, i_{y}, i_{z}}(t)$ denotes the noise term, $\lambda$ is the wavelength, $j=\sqrt{-1}$, and three electric angles $\theta_{k}, \varphi_{k}$, and $\phi_{k}$ [15], which are the functions of $\alpha_{k}$ and $\beta_{k}$, are, respectively, defined as $\theta_{k}=-2 \pi d \cos \beta_{k} \cos \alpha_{k} / \lambda$, $\varphi_{k}=-2 \pi d \cos \beta_{k} \sin \alpha_{k} / \lambda$, and $\phi_{k}=-2 \pi d \sin \beta_{k} / \lambda$. Since $\left(i_{x}, i_{y}, i_{z}\right) \in\{(1,0,0),(0,0,0),(0,1,0), \ldots,(1,0, M-$ $1),(0,0, M-1),(0,1, M-1)\}$, the vector form of (1) can be written as the following form:

$$
\mathbf{x}(t)=\mathbf{A s}(t)+\mathbf{n}(t) \quad t=1,2, \ldots, T,
$$

where $\mathbf{x}(t)=\left[x_{1,0,0}(t), x_{0,0,0}(t), x_{0,1,0}(t), \ldots, x_{1,0, M-1}(t)\right.$, $\left.x_{0,0, M-1}(t), x_{0,1, M-1}(t)\right]^{T}$ and $\mathbf{n}(t)=\left[n_{1,0,0}(t), n_{0,0,0}(t)\right.$, $\left.n_{0,1,0}(t), \ldots, n_{1,0, M-1}(t), n_{0,0, M-1}(t), n_{0,1, M-1}(t)\right]^{T}$ are $3 M \times 1$ 
vectors, $\mathbf{s}(t)=\left[s_{1}, s_{2}, \ldots, s_{K}\right]^{T}, \mathbf{A}=\left[\mathbf{a}\left(\theta_{1}, \varphi_{1}, \phi_{1}\right), \mathbf{a}\left(\theta_{2}\right.\right.$, $\left.\left.\varphi_{2}, \phi_{2}\right), \ldots, \mathbf{a}\left(\theta_{K}, \varphi_{K}, \phi_{K}\right)\right]$ is a $3 M \times K$ manifold matrix, and $\mathbf{a}\left(\theta_{k}, \varphi_{k}, \phi_{k}\right)=\left[e^{j \theta_{k}}, 1, e^{j \varphi_{k}}, e^{j \theta_{k}} e^{j \phi_{k}}, \ldots, e^{j \theta_{k}} e^{j(M-1) \phi_{k}}\right.$, $\left.e^{j(M-1) \phi_{k}}, e^{j \varphi_{k}} e^{j(M-1) \phi_{k}}\right]^{T}$ is a $3 M \times 1$ steering vector. By the properties of $\otimes$ and $\odot$, the steering vector $\mathbf{a}\left(\theta_{k}, \varphi_{k}, \phi_{k}\right)$ can be simplified to

$$
\begin{aligned}
\mathbf{a}\left(\theta_{k}, \varphi_{k}, \phi_{k}\right) & =\widetilde{\mathbf{a}}\left(\phi_{k}\right) \otimes\left[\breve{\mathbf{a}}\left(\theta_{k}\right) \odot \widehat{\mathbf{a}}\left(\varphi_{k}\right)\right] \\
& =\widetilde{\mathbf{a}}\left(\phi_{k}\right) \otimes \widehat{\mathbf{a}}\left(\theta_{k}, \varphi_{k}\right),
\end{aligned}
$$

where $\breve{\mathbf{a}}\left(\theta_{k}\right)=\left[e^{j \theta_{k}}, 1,1\right]^{T}, \widehat{\mathbf{a}}\left(\varphi_{k}\right)=\left[1,1, e^{j \varphi_{k}}\right]^{T} \widehat{\mathbf{a}}\left(\theta_{k}, \varphi_{k}\right)=$ $\breve{\mathbf{a}}\left(\theta_{k}\right) \odot \widehat{\mathbf{a}}\left(\varphi_{k}\right)=\left[e^{j \theta_{k}}, 1, e^{j \varphi_{k}}\right]^{T}$, and $\widetilde{\mathbf{a}}\left(\phi_{k}\right)=\left[1, e^{j \phi_{k}}, \ldots\right.$, $\left.e^{j(M-1) \phi_{k}}\right]^{T}$. Denote $\widetilde{\mathbf{A}}(\phi)=\left[\widetilde{\mathbf{a}}\left(\phi_{1}\right), \widetilde{\mathbf{a}}\left(\phi_{2}\right), \ldots, \widetilde{\mathbf{a}}\left(\phi_{K}\right)\right]$ and $\widehat{\mathbf{A}}(\theta, \varphi)=\left[\widehat{\mathbf{a}}\left(\theta_{1}, \varphi_{1}\right), \widehat{\mathbf{a}}\left(\theta_{2}, \varphi_{2}\right), \ldots, \widehat{\mathbf{a}}\left(\theta_{K}, \varphi_{K}\right)\right]$ as the manifold matrices that contain information about $\phi$ and $(\theta, \varphi)$, respectively. The purpose of this simplification is to construct two corresponding noise subspaces to estimate the electric angles. Then, the matrix form of (2) is given by

$$
\widetilde{\mathbf{X}}(t)=\operatorname{unvec}(\mathbf{x}(t))=\widetilde{\mathbf{A}}(\phi) \mathbf{G}(t)+\widetilde{\mathbf{N}}(t)
$$

$$
t=1,2, \ldots, T
$$

where $\mathbf{G}(t)=\left[s_{1} \widehat{\mathbf{a}}\left(\theta_{1}, \varphi_{1}\right), s_{2} \widehat{\mathbf{a}}\left(\theta_{2}, \varphi_{2}\right), \ldots, s_{K} \widehat{\mathbf{a}}\left(\theta_{K}, \varphi_{K}\right)\right]^{T}$ is a $K \times 3$ source matrix and $\widetilde{\mathbf{X}}(t)$ and $\widetilde{\mathbf{N}}(t)$ are $M \times 3$ matrices whose elements in the $(m, n)$ th entry are the $(3 m+n-3)$ th elements of vectors $\mathbf{x}(t)$ and $\mathbf{n}(t)$, respectively. It is easy to see from (4) that $\phi$ and $(\theta, \varphi)$ span the column and row spaces of $\widetilde{\mathbf{x}}(t)$, respectively, and the manifold matrix $\widetilde{\mathbf{A}}(\phi)$ is determined by $\phi$ while the sources matrix $\mathbf{G}(t)$ is decided by $(\theta, \varphi)$. The electric angles estimation can be performed by estimating $\phi$ and $(\theta, \varphi)$ successively instead of jointly estimating them. Since $\phi$ is separated from $(\theta, \varphi), \phi$ can be firstly estimated and then $(\theta, \varphi)$ can be obtained in terms of the estimates of $\phi$.

Let $\left\{\widehat{\phi}_{p}\right\}_{p=1}^{P_{\phi}}$ be a sampling grid which covers the entire spatial domain so that the true electric angles $\left\{\phi_{k}\right\}_{k=1}^{K}$ are on the sampling grid set where $P_{\phi}\left(P_{\phi} \gg K\right)$ denotes the number of the sampling grid. This means that if $\widehat{\phi}_{p_{1}}, \widehat{\phi}_{p_{2}}, \ldots, \widehat{\phi}_{p_{K}}$ are one to one corresponding to the true electric angles $\phi_{1}, \phi_{2}, \ldots, \phi_{K}$, we have

$$
g_{p}= \begin{cases}s_{k} & p=p_{k}(k=1,2, \ldots, K) \\ 0 & \text { elsewhere. }\end{cases}
$$

By denoting $\overline{\mathbf{X}} \triangleq[\widetilde{\mathbf{X}}(1), \widetilde{\mathbf{X}}(2), \ldots, \widetilde{\mathbf{X}}(T)]$ as $M \times 3 T$ data matrix, (4) can be rewritten as the following sparse form:

$$
\begin{aligned}
\overline{\mathbf{X}} & =\overline{\mathbf{A}}(\phi) \overline{\mathbf{G}}+[\widetilde{\mathbf{N}}(1), \widetilde{\mathbf{N}}(2), \ldots, \widetilde{\mathbf{N}}(T)] \\
& =\overline{\mathbf{A}}(\phi) \overline{\mathbf{G}}+\overline{\mathbf{N}},
\end{aligned}
$$

where $\overline{\mathbf{A}}(\phi)=\left[\widetilde{\mathbf{a}}\left(\phi_{1}\right), \widetilde{\mathbf{a}}\left(\phi_{2}\right), \ldots, \widetilde{\mathbf{a}}\left(\phi_{P_{\phi}}\right)\right]$ is the $M \times P_{\phi}$ manifold matrix corresponding to all potential electric angles which is also defined as an overcomplete dictionary in CS. Since $\left\{\overline{\mathbf{G}}_{t}\right\}_{t=1}^{3 T}$ share the same support based on joint sparsity,
$\overline{\mathbf{G}}=\left[\overline{\mathbf{G}}_{1}, \overline{\mathbf{G}}_{2}, \ldots, \overline{\mathbf{G}}_{P_{\phi}}\right]^{T}$ has $K$ nonzero rows, that is, the row $K$-sparse, where $\overline{\mathbf{G}}_{p_{\phi}}=\left[g_{p_{\phi}}(1) \widehat{\mathbf{A}}_{k}^{T}(\theta, \varphi), g_{p_{\phi}}(2) \widehat{\mathbf{A}}_{k}^{T}(\theta, \varphi), \ldots\right.$, $\left.g_{p_{\phi}}(T) \widehat{\mathbf{A}}_{k}^{T}(\theta, \varphi)\right]^{T}$ is a $3 T \times 1$ vector. To estimate the electric angle, the support needs be determined by the matrix $\mathbf{X}$ which is given by

$$
\mathbf{X}=\boldsymbol{\Phi} \overline{\mathbf{A}}(\phi) \overline{\mathbf{G}}+\boldsymbol{\Phi} \overline{\mathbf{N}}=\widehat{\mathbf{A}}(\phi) \overline{\mathbf{G}}+\mathbf{N},
$$

where $\Phi$ is the $N \times M$ measurement matrix and $N$ is the number of nonadaptive linear projection measurements.

\section{DOA Estimation}

In this section, a 2D DOA estimation problem is solved by two steps in the CS scenario. Based on the above source model, a 1D DOA estimation is firstly performed to estimate $\phi$ and get the information about $(\theta, \varphi)$ which is contained in the source matrix of (7). Secondly, based on the estimates of $\phi$, two electric angles $\theta$ and $\varphi$ can be estimated by minimizing the relaxation of the residual fitting error [19]

$$
J^{(p, q)}(\mathbf{Y})=\min \sum_{i=1}^{P}\left(\sum_{j=1}^{Q}\left|y_{i j}\right|^{q}\right)^{p / q}
$$

where $\mathbf{Y} \in \mathbb{C}^{P \times Q}$ is assumed to be the residual fitting error matrix. For simplicity, the case $q=2$ is considered in the rest of this paper; that is,

$$
J^{(p, 2)}(\mathbf{Y})=\min \sum_{i=1}^{P}\left(\sum_{j=1}^{Q}\left|y_{i j}\right|^{2}\right)^{p / 2} .
$$

The motivation to choose $q=2$ is the following two main reasons. As can be seen, the first reason is that the number of nonzero rows can be provided as $p$ approaches zero. A nonzero row can serve as a penalty factor with the reduction of $p$ which promotes a sparse frame among all rows. Secondly, practical issues such as computational complexity are also in favor of this choice. In the case $q=2$, a low computational complexity is obtained by minimizing (9) instead of (8) [20]. Then, for notational convenience, we denote $J^{(p, 2)}(\mathbf{Y})$ by $J^{(p)}(\mathbf{Y})$ which is called $l_{p}$-norm.

\section{1. $C S-R D-l_{p}-M U S I C$}

Step 1 (estimate $\phi$ by CS-MUSIC). CS-MUSIC, which is an extension of MUSIC, can identify the parts of support using CS-based methods, after which the remaining supports are estimated by the generalized MUSIC criterion. The main contribution of CS-MUSIC is to overcome the error caused by losing rank which may cause disastrous consequences in conventional MUSIC.

Let $\operatorname{supp} \overline{\mathbf{G}}=\left\{1 \leq g \leq P_{\phi}: \overline{\mathbf{G}}^{\mathfrak{g}} \neq \mathbf{0}\right\}$ and $R(\widehat{\mathbf{A}}(\phi))$ denote the support of $\overline{\mathbf{G}}$ and the range space of $\widehat{\mathbf{A}}(\phi)$, respectively. Due to (4), the number of snapshots is extended to $3 T$ which is one of the advantages of the proposed method. 
It is obvious that CS-MUSIC can be simplified as MUSIC for $K \leq 3 T$. However, CS-MUSIC can estimate DOA with success but MUSIC fails in the $K>3 T$ case. In CS-MUSIC, if $K>3 T, K-3 T$ indices of supp $\overline{\mathbf{G}}$ are determined by CSbased methods such as simultaneous orthogonal matching pursuit (SOPM) [21] so that column vector space $R\left(\widehat{\mathbf{A}}_{\eta_{K-3 T}}(\phi)\right)$ is decided, where $\eta_{K-3 T}$ is the set of indices and $\overline{\mathbf{A}}_{\eta_{K-3 T}}(\phi)$ is the submatrix of $\widehat{\mathbf{A}}(\phi)$ with columns indexed by $\eta_{K-3 T}$. The remaining $3 T$ indices of supp $\overline{\mathbf{G}}$ are obtained by the generalized MUSIC criterion. To make CS-MUSIC applicable for all range of the snapshot, two new orthogonal spaces $R\left(\widehat{\mathbf{A}}_{\overline{\mathbf{G}}}\right)$ and $R\left(P_{R(\mathbf{Q})}-P_{R(\mathbf{Q Q}}{ }^{H} \widehat{\mathbf{A}}_{\left.\eta_{K-3 T}(\phi)\right)}\right)$ are constructed where $P_{R(\mathrm{Q})}$ is the orthogonal projection onto the noise subspace $R(\mathbf{Q})$. Then, the electric angle $\phi$ can be estimated by the spectrum search. Now, the major steps of CS-MUSIC are summarized as follows.

(1) Find $K-3 T$ indices of supp $\overline{\mathbf{G}}$ by SOMP and let $\eta_{K-3 T}$ be the set of indices.

(2) Determine $\widehat{\mathbf{A}}_{\eta_{K-3 T}}(\phi)$ in terms of $\eta_{K-3 T}$ and construct the noise subspace of CS-MUSIC $R\left(P_{R(\mathbf{Q})}-\right.$ $\left.P_{R(\mathbf{Q Q}}^{H} \widehat{\mathbf{A}}_{\eta_{K-3 T}}(\phi)\right)$.

(3) For $i \in\left\{1,2, \ldots, P_{\phi}\right\}$, calculate the spatial spectrum, $\left.P_{\text {spectral }}=1 / \widehat{\mathbf{A}}_{i}^{H}(\phi)\left[P_{R(\mathbf{Q})}-P_{R(\mathbf{Q Q}} \overline{\mathbf{A}}_{\eta_{K-3 T}}(\phi)\right)\right] \widehat{\mathbf{A}}_{i}(\phi)$, and then estimate $\phi$ by the locations of $K$ highest peaks of the spatial spectrum.

Step 2 (estimate $\theta$ and $\varphi$ by RD- $l_{p}$-MUSIC). In this step, RD$l_{p}$-MUSIC is exploited to estimate electric angles $\theta$ and $\varphi$ in terms of the estimates of $\phi$. Let $\left\{\bar{\phi}_{1}, \bar{\phi}_{2}, \ldots, \bar{\phi}_{K}\right\}$ denote the locations of $K$ highest peaks. Then, the row number corresponding to locations of $K$ highest peaks is denoted as $\left\{i_{1}, i_{2}, \ldots, i_{K}\right\}$ and these rows contain information about $(\theta, \varphi)$. It is clear that the $i$ th row vector of source matrix $\left[\widehat{g}_{1,1}(i), \widehat{g}_{2,1}(i), \ldots, \widehat{g}_{T, 1}(i), \ldots, \widehat{g}_{1,3}(i), \widehat{g}_{2,3}(i), \ldots, \widehat{g}_{T, 3}(i)\right], i=$ $i_{1}, i_{2}, \ldots, i_{K}$, is a $1 \times 3 T$ vector and thus the matrix form $\widehat{\mathbf{G}}(i)$ is a $T \times 3$ matrix whose element in the $(m, n)$ th entry is $\widehat{g}_{m, n}(i)$. Denote $\underset{\mathbf{A}}{\mathbf{A}}(\theta, \varphi)=\widehat{\mathbf{A}}^{T}(\theta, \varphi)$ and $\widehat{\mathbf{S}}=[\mathbf{s}(1), \mathbf{s}(2), \ldots, \mathbf{s}(T)]^{T}$ so that the $\widetilde{l}_{p}$-norm minimization problem can be expressed as

$$
\begin{aligned}
& J^{(p)}(\theta, \varphi)=\min _{\theta, \varphi} J^{(p)}(\widehat{\mathbf{G}}(i)-\widehat{\mathbf{S}} \underset{\mathbf{A}}{\mathbf{A}}(\theta, \varphi)) \\
&=\min _{\theta, \varphi} \sum_{t=1}^{T}\left(\left\|\widehat{\mathbf{G}}^{t}(i)-\widehat{\mathbf{S}}^{t} \underset{\sim}{\mathbf{A}}(\theta, \varphi)\right\|_{2}\right)^{p} \\
& \\
& \quad i=i_{1}, i_{2}, \ldots, i_{K} .
\end{aligned}
$$

Note that $K l_{p}$-norm minimization problems have the same structure and can be solved in a similar manner. Hence, we only need to find the optimal solution of one minimization problem. The remaining $K-1$ minimization problems can be handled in the same way.

Since the objective function (10) requires an exhaustive $2 \mathrm{D}$ search, high computational cost is needed for precise estimation which can result in the reduction of algorithmic efficiency. To avoid heavy computational load, RD- $l_{p}$-MUSIC is proposed for $2 \mathrm{D}$ estimation just through $1 \mathrm{D}$ search. A detailed derivation process of exploiting RD- $l_{p}$-MUSIC for estimating $\theta$ and $\varphi$ is given as follows.

Denote $F(\theta, \varphi)=\left\|\widehat{\mathbf{G}}^{t}(i)-\widehat{\mathbf{S}}^{t} \underset{\mathbf{A}}{\mathbf{A}}(\theta, \varphi)\right\|_{2}^{2}$ so that RD can be realized by minimizing $F(\theta, \varphi)$. By the property of $\|\cdot\|_{2}$, we have

$$
\begin{aligned}
& F(\theta, \varphi) \\
& =\left[\widehat{\mathbf{G}}^{t}(i)-\widehat{\mathbf{S}}^{t} \underline{\mathbf{A}}(\theta, \varphi)\right]\left[\widehat{\mathbf{G}}^{t}(i)-\widehat{\mathbf{S}}^{t} \underline{\mathbf{A}}(\theta, \varphi)\right]^{H} \\
& =\text { const } \\
& +\widehat{\mathbf{S}}^{t}[\underline{\mathbf{A}}(\theta) \odot \underbrace{\mathbf{A}}(\varphi)][\underline{\mathbf{A}}(\theta) \odot \underset{\sim}{\mathbf{A}}(\varphi)]^{H}\left(\widehat{\mathbf{S}}^{t}\right)^{H} \\
& -2 \operatorname{Re}\left\{\widehat{\mathbf{S}}^{t}[\underbrace{\mathbf{A}}(\theta) \odot \underbrace{\mathbf{A}}(\varphi)]\left(\widehat{\mathbf{G}}^{t}(i)\right)^{H}\right\},
\end{aligned}
$$

where $\underline{\mathbf{A}}(\theta)=\left[\breve{\mathbf{a}}\left(\theta_{1}\right), \breve{\mathbf{a}}\left(\theta_{2}\right), \ldots, \breve{\mathbf{a}}\left(\theta_{K}\right)\right]^{T}$ and $\underline{\mathbf{A}}(\varphi)=\left[\widehat{\mathbf{a}}\left(\varphi_{1}\right)\right.$, $\left.\widehat{\mathbf{a}}\left(\varphi_{2}\right), \ldots, \widehat{\mathbf{a}}\left(\varphi_{K}\right)\right]^{T}$. Denote that $\mathbf{P}_{(K)}=\left[\mathbf{E}_{11}^{(K)}, \mathbf{E}_{22}^{(K)}, \ldots, \mathbf{E}_{K K}^{(K)}\right]^{T}$ and $\mathbf{P}_{(3)}=\left[\mathbf{E}_{11}^{(3)}, \mathbf{E}_{22}^{(3)}, \mathbf{E}_{33}^{(3)}\right]^{T}$ are $K^{2} \times K$ and $9 \times 3$ selection matrices, respectively, so that the following equation holds based on selection matrices [22]:

$$
\begin{aligned}
& \underbrace{\mathbf{A}}(\theta) \odot \underbrace{\mathbf{A}}_{(K)}(\varphi) \\
& \quad=\mathbf{P}_{(K)}^{T}[\theta) \otimes \underbrace{\mathbf{A}}(\varphi)] \mathbf{P}_{(3)} \\
& \quad=\mathbf{P}_{(K)}^{T}[\underbrace{\mathbf{A}}(\theta) \otimes \mathbf{I}_{K \times K}][\mathbf{I}_{3 \times 3} \otimes \underbrace{\mathbf{A}}(\varphi)] \mathbf{P}_{(3)} .
\end{aligned}
$$

During the aforesaid process, selection matrices are of the greatest importance. The purpose of utilizing selection matrices is to separate $\theta$ from $\varphi$ and reduce dimension. Moreover, based on the following equation,

$$
\mathbf{Q}_{1}\left[\mathbf{I}_{3 \times 3} \otimes \underset{\mathbf{A}}{\mathbf{A}}(\varphi)\right] \mathbf{Q}_{2}=\underbrace{}_{\mathbf{A}}(\varphi),
$$

where $\mathbf{Q}_{1}=\left[\begin{array}{ll}\mathbf{I}_{K \times K} & \mathbf{0}_{K \times 2 K}\end{array}\right]$ and $\mathbf{Q}_{2}=\left[\begin{array}{ll}\mathbf{I}_{3 \times 3} & \mathbf{0}_{3 \times 6}\end{array}\right]^{T}$, (12) can be further rewritten as

$$
\begin{aligned}
& \underbrace{\mathbf{A}}(\theta) \odot \underset{\sim}{\mathbf{A}}(\varphi) \\
& \quad=\mathbf{P}_{(K)}^{T}\left[\underline{\mathbf{A}}(\theta) \otimes \mathbf{I}_{K \times K}\right] \mathbf{Q}_{1}^{-1} \stackrel{\mathbf{A}}{ }(\varphi) \mathbf{Q}_{2}^{-1} \mathbf{P}_{(3)} .
\end{aligned}
$$

Subsequently, by substituting (14) into (11), we have

$$
\begin{gathered}
F(\theta, \varphi)=\text { const }-2 \operatorname{Re}\left\{\widehat{\mathbf{S}}^{t} \mathbf{P}_{(K)}^{T}[\underbrace{\mathbf{A}}(\theta) \otimes \mathbf{I}_{K \times K}]\right. \\
\left.\cdot \mathbf{Q}_{1}^{-1} \underset{\mathbf{A}}{\mathbf{A}}(\varphi) \mathbf{Q}_{2}^{-1} \mathbf{P}_{(3)}\left(\widehat{\mathbf{G}}^{t}(i)\right)^{H}\right\}+\widehat{\mathbf{S}}^{t} \mathbf{P}_{(K)}^{T}[\underbrace{\mathbf{A}}(\theta) \\
\left.\otimes \mathbf{I}_{K \times K}\right] \mathbf{Q}_{1}^{-1} \underline{\mathbf{A}}(\varphi) \mathbf{Q}_{2}^{-1} \mathbf{P}_{(3)} \mathbf{P}_{(3)}^{H}\left(\mathbf{Q}_{2}^{-1}\right)^{H} \underline{\mathbf{A}}^{H}(\varphi) \\
\cdot\left(\mathbf{Q}_{1}^{-1}\right)^{H}[\underbrace{\mathbf{A}^{H}}(\theta) \otimes \mathbf{I}_{K \times K}]\left(\mathbf{P}_{(K)}^{T}\right)^{H}\left(\widehat{\mathbf{S}}^{t}\right)^{H} .
\end{gathered}
$$

To eliminate trivial solutions $\underbrace{\mathbf{A}}(\theta)=\mathbf{0}_{K \times 3}$ and $\underset{\sim}{\mathbf{A}}(\varphi)=$

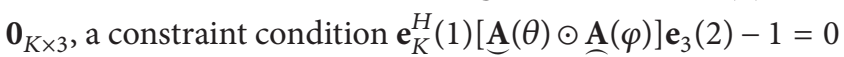


is considered. Hence, the cost function $L(\theta, \varphi)$ is given as the following form:

$$
\begin{aligned}
& L(\theta, \varphi)=\widehat{\mathbf{S}}^{t} \mathbf{P}_{(K)}^{T}\left[\underline{\mathbf{A}}(\theta) \otimes \mathbf{I}_{K \times K}\right] \mathbf{Q}_{1}^{-1} \underline{\mathbf{A}}(\varphi) \\
& \cdot \mathbf{Q}_{2}^{-1} \mathbf{P}_{(3)} \mathbf{P}_{(3)}^{H}\left(\mathbf{Q}_{2}^{-1}\right)^{H} \underbrace{\mathbf{A}^{H}}(\varphi)\left(\mathbf{Q}_{1}^{-1}\right)^{H}[\underbrace{\mathbf{A}^{H}}(\theta) \\
& \left.\otimes \mathbf{I}_{K \times K}\right]\left(\mathbf{P}_{(K)}^{T}\right)^{H}\left(\widehat{\mathbf{s}}^{t}\right)^{H} \\
& -2 \operatorname{Re}\left\{\widehat{\mathbf{S}}^{t} \mathbf{P}_{(K)}^{T}\left[\underline{\mathbf{A}}(\theta) \otimes \mathbf{I}_{K \times K}\right] \mathbf{Q}_{1}^{-1} \stackrel{\mathbf{A}}{ }(\varphi)\right. \\
& \left.\cdot \mathbf{Q}_{2}^{-1} \mathbf{P}_{(3)}\left(\overline{\mathbf{G}}^{t}(i)\right)^{H}\right\}-\mu\left[\mathbf{e}_{K}^{H}(1)\right. \\
& \cdot \mathbf{P}_{(K)}^{T}[\underbrace{\mathbf{A}}(\theta) \otimes \mathbf{I}_{K \times K}] \mathbf{Q}_{1}^{-1} \underbrace{\mathbf{A}(}_{(\varphi)} \mathbf{Q}_{2}^{-1} \mathbf{P}_{(3)} \mathbf{e}_{3}(2)-1],
\end{aligned}
$$

where $\mu$ is a constant. By setting the partial derivation of (16) with respect to $\mathbf{A}(\varphi)$ to zero, we have

$$
\begin{aligned}
\frac{\partial L(\theta, \varphi)}{\partial \mathcal{A}_{(\varphi)}(\varphi)} & 2 \mathbf{W}\left(\widehat{\mathbf{S}}^{t}\right)^{H} \widehat{\mathbf{S}}^{t} \mathbf{W} \underset{-}{\mathbf{A}}(\varphi) \mathbf{V V}^{H} \\
& -2 \operatorname{Re}\left\{\mathbf{W}^{H}\left(\widehat{\mathbf{S}}^{t}\right)^{H}\left(\widehat{\mathbf{G}}^{t}(i)\right)^{H} \mathbf{V}^{H}\right\} \\
& -\mu\left[\mathbf{W}^{H} \mathbf{e}_{K}(1) \mathbf{e}_{3}^{H}(2) \mathbf{V}^{H}\right]=\mathbf{0},
\end{aligned}
$$

where $\mathbf{W}=\mathbf{P}_{(K)}^{T}\left[\underline{\mathbf{A}}(\theta) \otimes \mathbf{I}_{K \times K}\right] \mathbf{Q}_{1}^{-1}$ and $\mathbf{V}=\mathbf{Q}_{2}^{-1} \mathbf{P}_{(3)}$. By (17) and the constraint condition $\mathbf{e}_{K}^{H}(1)[\underline{\mathbf{A}}(\theta) \odot \underbrace{\mathbf{A}}_{(\varphi)}(\varphi) \mathbf{e}_{3}(2)=1, \mu$ can be expressed as

$$
\mu=\frac{2-2 \mathbf{e}_{K}^{H}(1)\left(\widehat{\mathbf{S}}^{t}\right)^{-1} \operatorname{Re}\left\{\widehat{\mathbf{G}}^{t}(i)\right\} \mathbf{e}_{3}(2)}{\mathbf{e}_{K}^{H}(1)\left(\widehat{\mathbf{S}}^{t}\right)^{-1}\left[\left(\widehat{\mathbf{S}}^{t}\right)^{-1}\right]^{H} \mathbf{e}_{K}(1)} .
$$

Therefore, it can be deduced from (17) and (18) that

$$
\begin{aligned}
& \underset{\sim}{\mathbf{A}(\varphi)}=\mathbf{W}^{-1}\left(\widehat{\mathbf{S}}^{t}\right)^{-1}\left[\operatorname{Re}\left\{\widehat{\mathbf{G}}^{t}(i)\right\}\right. \\
& \left.+\frac{\left[1-\mathbf{e}_{K}^{H}(1)\left(\widehat{\mathbf{G}}^{t}(i)\right)^{-1} \operatorname{Re}\left\{\widehat{\mathbf{G}}^{t}(i)\right\} \mathbf{e}_{3}(2)\right] \mathbf{e}_{K}(1) \mathbf{e}_{3}^{H}(2)}{\mathbf{e}_{K}^{H}(1)\left(\widehat{\mathbf{S}}^{t}\right)^{-1}\left[\left(\widehat{\mathbf{S}}^{t}\right)^{-1}\right]^{H} \mathbf{e}_{K}(1)}\right] \\
& \cdot \mathbf{V}^{-1} .
\end{aligned}
$$

As one may note, $\mathbf{A}(\theta, \varphi)$ is transformed into $\widehat{\mathbf{U}}(\theta)$ by inserting $\mathbf{A}(\varphi)$ into $\underset{\mathbf{A}}{\mathbf{A}}, \varphi)$ and thus $\mathrm{RD}$ is realized so that 2D DOA estimation just requires 1D search. Furthermore, $\mathrm{RD}$ can avoid the failure occurrence in separating $\theta$ from $\varphi$. As a matter of fact, $\mathrm{RD}$ also provides an important clue for expounding the relationship between $\theta$ and $\varphi$. Therefore, RD is the foundation of estimating electric angles $\theta$ and $\varphi$ by RD$l_{p}$-MUSIC. Then, we focus on the solver for the last remaining problem to estimate $\theta$; that is,

$$
J^{(p)}(\theta)=\min _{\theta} \sum_{t=1}^{T}\left(\left\|\widehat{\mathbf{G}}^{t}(i)-\widehat{\mathbf{S}}^{t} \widehat{\mathbf{U}}(\theta)\right\|_{2}\right)^{p} .
$$

By denoting $\mathbf{r}^{t}=\widehat{\mathbf{G}}^{t}(i)-\widehat{\mathbf{S}}^{t} \widehat{\mathbf{U}}(\theta)$ as the residual vector, the objective function $f\left(r_{i}^{t}\right)$ can be expressed as

$$
f\left(r_{i}^{t}\right)=\sum_{t=1}^{T}\left(\sum_{i=1}^{3}\left|r_{i}^{t}\right|^{2}\right)^{p / 2} .
$$

Based on the following equation,

$$
\frac{\partial f}{\partial\left(r_{i}^{t}\right)^{*}}=\frac{1}{2}\left(\frac{\partial f}{\partial \operatorname{Re}\left\{r_{i}^{t}\right\}}+j \frac{\partial f}{\partial \operatorname{Im}\left\{r_{i}^{t}\right\}}\right)
$$

the partial derivative with respect to $\left(r_{i}^{t}\right)^{*}$ can be given by

$$
\frac{\partial f}{\partial\left(r_{i}^{t}\right)^{*}}=\frac{p}{2}\left(\sum_{t=1}^{T}\left|r_{i}^{t}\right|^{2}\right)^{p / 2-1} r_{i}^{t} .
$$
by

Due to (23), the gradient of $f$ with respect to $\mathbf{r}^{t}$ is given

$$
\frac{\partial f}{\partial \mathbf{r}^{t}}=\left[\frac{\partial f}{\partial\left(r_{1}^{t}\right)^{*}} \frac{\partial f}{\partial\left(r_{2}^{t}\right)^{*}} \frac{\partial f}{\partial\left(r_{3}^{t}\right)^{*}}\right]=\frac{p}{2} \mathbf{r}^{t} \odot \boldsymbol{\Lambda},
$$

where

$$
\begin{aligned}
& \boldsymbol{\Lambda} \\
& =\left[\left(\sum_{t=1}^{T}\left|r_{1}^{t}\right|^{2}\right)^{p / 2-1}\left(\sum_{t=1}^{T}\left|r_{2}^{t}\right|^{2}\right)^{p / 2-1}\left(\sum_{t=1}^{T}\left|r_{3}^{t}\right|^{2}\right)^{p / 2-1}\right] .
\end{aligned}
$$

Denote $\mathbf{F}=\operatorname{diag}(\boldsymbol{\Lambda})$ so that $(24)$ can be rewritten as

$$
\frac{\partial f}{\partial \mathbf{r}^{t}}=\frac{p}{2} \mathbf{r}^{t} \mathbf{F}
$$

Note that $\mathbf{F}$ is a positive definite matrix clearly. Then, since the second-order partial derivative of $f$ with respect to $r_{i}^{t}$ is given as the following form,

$$
\begin{aligned}
& \frac{\partial^{2} f}{\partial\left(r_{i}^{t}\right)^{T} \partial\left(r_{j}^{t}\right)^{*}} \\
& = \begin{cases}\frac{p}{2}\left(\sum_{t=1}^{T}\left|r_{i}^{t}\right|^{2}\right)^{p / 2-1}\left[\left(\frac{p}{2}-1\right)\left(\sum_{i=1}^{3}\left|r_{i}^{t}\right|^{2}\right)^{-1}\left|r_{i}^{t}\right|^{2}+1\right] \begin{array}{l}
i=j \\
0
\end{array} \\
i \neq j,\end{cases}
\end{aligned}
$$

the $3 \times 3$ Hessian matrix of $f$ with respect to $\mathbf{r}^{t}$ is given by

$$
\begin{aligned}
& \mathbf{H}_{\mathbf{r}^{t}\left(\mathbf{r}^{t}\right)^{*}}=\frac{\partial^{2} f}{\partial\left(\mathbf{r}^{t}\right)^{T} \partial\left(\mathbf{r}^{t}\right)^{*}}=\frac{p}{2} \\
& \cdot \mathbf{F}\left[\left(\frac{p}{2}-1\right)\left(\sum_{i=1}^{3}\left|r_{i}^{t}\right|^{2}\right)^{-1} \operatorname{diag}\left(\left(\mathbf{r}^{t}\right)^{T} \mathbf{r}^{t}\right)\right. \\
& \left.+\mathbf{I}_{3 \times 3}\right] .
\end{aligned}
$$


Denote the gradient of $f$ with respect to $\widehat{\mathbf{U}}(\theta)$ as $\Gamma=$ $\left[\boldsymbol{\Gamma}_{1}, \boldsymbol{\Gamma}_{2}, \boldsymbol{\Gamma}_{3}\right]$ so that $\boldsymbol{\Gamma}_{i}$ is computed as

$$
\boldsymbol{\Gamma}_{i}=\frac{\partial f}{\partial \widehat{\mathbf{U}}_{i}^{*}}=-\left[\left(\widehat{\mathbf{S}}^{t}\right)^{T} \otimes \mathbf{e}_{3}^{T}(i)\right] \frac{p}{2}\left(\mathbf{r}^{t} \mathbf{F}\right)^{T} .
$$

Then, the Hessian matrix of $f$ with respect to $\widehat{\mathbf{U}}(\theta)$ is

$$
\begin{aligned}
\mathbf{H}_{\widehat{\mathbf{U}}^{*} \widehat{\mathbf{U}}} & =\frac{\partial^{2} f}{\partial \widehat{\mathbf{U}}^{*} \partial \widehat{\mathbf{U}}^{T}} \\
& =\left[\left(\widehat{\mathbf{S}}^{t}\right)^{T} \otimes \mathbf{\Sigma}\right] \mathbf{P}_{(3)} \mathbf{H}_{\mathbf{r}^{t}\left(\mathbf{r}^{t}\right)^{*}} \mathbf{P}_{(3)}^{T}\left[\left(\widehat{\mathbf{S}}^{t}\right)^{*} \otimes \Sigma^{T}\right],
\end{aligned}
$$

where $\boldsymbol{\Sigma}=\left[\begin{array}{lll}\mathbf{e}_{3}(1) & \mathbf{e}_{3}(2) & \mathbf{e}_{3}(3)\end{array}\right]$. By applying the Newton method, the following sequence $\left\{\widehat{\mathbf{U}}^{(z)}(\theta)\right\}, z=0,1,2, \ldots$, is obtained to find the minimization of $f$ :

$$
\widehat{\mathbf{U}}^{(z+1)}(\theta)=\widehat{\mathbf{U}}^{(z)}(\theta)+\sigma_{z} \Delta^{(z)}\left(\widehat{\mathbf{U}}^{(z)}(\theta)\right),
$$

where $\sigma_{z}$ is a positive step and $\Delta^{(z)}\left(\widehat{\mathbf{U}}^{(z)}(\theta)\right)=$ $-\mathbf{H}_{\widehat{\mathbf{U}}^{*} \widehat{\mathbf{U}}} \boldsymbol{\Gamma}\left(\widetilde{\mathbf{U}}^{(z)}(\theta)\right)$. The initial value of $\widehat{\mathbf{U}}^{(z)}(\theta)$ is defined as $\widehat{\mathbf{U}}^{(0)}(\theta)=\left[\left(\widehat{\mathbf{S}}^{t}\right)^{H} \widehat{\mathbf{S}}^{t}\right]^{-1}\left(\widehat{\mathbf{S}}^{t}\right)^{H} \widehat{\mathbf{G}}^{t}(i)$. Specifically, the iteration is terminated if the following stopping criterion is satisfied:

$$
\frac{J^{(p)}\left(\theta^{(z)}\right)-J^{(p)}\left(\theta^{(z+1)}\right)}{J^{(p)}\left(\theta^{(z)}\right)}<\varepsilon
$$

for some small $\varepsilon$. In the simulations, $\varepsilon$ is set to $10^{-7}$. After determining $\widehat{\mathbf{U}}(\theta)$, the projection matrix onto the noise subspace is given by

$$
\widehat{\mathbf{P}}=\mathbf{I}_{3 \times 3}-\mathbf{U}(\theta)\left[\mathbf{U}^{H}(\theta) \mathbf{U}(\theta)\right]^{-1} \mathbf{U}^{H}(\theta),
$$

where $\mathbf{U}(\theta)=\widehat{\mathbf{U}}^{H}(\theta)$. Then, by computing the spatial spectrum, $P_{\text {spetral }}(\theta)=1 / \breve{\mathbf{a}}^{H}(\theta) \widehat{\mathbf{P}} \breve{a}(\theta)$, and searching its peaks, we can provide an accurate estimation for $\theta$. Once $\theta$ is obtained from the spatial spectrum, $\varphi$ is easily estimated in terms of (19). Therefore, RD- $l_{p}$-MUSIC avoids pair matching.

After electric angles are estimated, the azimuth angle $\alpha_{k}$ and elevation angle $\beta_{k}$ of the $k$ th source can be given by $\alpha_{k}=$ $\arctan \left(\varphi_{k} / \theta_{k}\right)$ and $\beta_{k}=\arctan \left(\phi_{k} \sin \alpha_{k} / \varphi_{k}\right)$, respectively. The major steps of RD- $l_{p}$-MUSIC for estimating $\theta$ and $\varphi$ are given as follows.

(1) The $l_{p}$-norm minimization problem (10) is given in terms of the estimates of $\phi$.

(2) Due to (18) and (19), calculate $\mu$ and $\mathbf{A}(\varphi)$ so that $\mathrm{RD}$ is realized.

(3) Calculate the gradient $\boldsymbol{\Gamma}$ and Hessian matrix $\mathbf{H}_{\widehat{U}^{*} \widehat{U}}$ in terms of (29) and (30). Then, update $\widehat{\mathbf{U}}(\theta)$ in terms of (31).

(4) If the stopping criterion (32) is satisfied, stop the update and go to the next step. Otherwise, return to step (3).

(5) Estimate $\theta$ by searching the spatial spectrum and then $\varphi$ is estimated in terms of (19).

(6) Estimate the azimuth and elevation angles according to the estimates of electric angles.
3.2. Discussion. Note that electric angle $\phi$ is estimated based

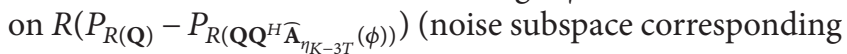
to $\phi)$ and the estimates of electric angles $\theta$ and $\varphi$ obtained by $R(\widehat{\mathbf{P}})$ (noise subspace corresponding to $\theta$ and $\varphi$ ) rely on the estimate quality of $\phi$. Moreover, the elevation angle is estimated based on the estimate of the azimuth angle and both of them use the arc-tangent operator. It is worth pointing out that since the arc-tangent is a one-to-one function and its argument is allowed to be larger than unity, the proposed method never fails. This is the major reason why the azimuth and elevation angles are estimated using three electric angles instead of two electric angles.

Regarding the proposed array configuration, we make full use of structural feature for 2D DOA estimation, which shows no failure in the entire ranges of $\alpha$ and $\beta$. Furthermore, when seen from above, the proposed array configuration seems to be an L-array configuration. Referring to [10], the CramerRao bound (CRB) based on the L-shaped array configuration is lower than those of other array configurations. Therefore, the proposed array configuration not only guarantees that the failure probability tends to zero, but it can also have high precision.

\section{Simulation Results}

In this section, several simulation results are presented to validate the superior performance of the proposed method as compared to PM of one L-shaped array configuration and 2D-MUSIC. The proposed method and 2D-MUSIC are performed in the proposed array configuration and PM is performed in the L-shaped array configuration of the $x$ $y$ plane. The total numbers of elements for array configurations and the spacing between the adjacent elements are set to 9 and $d=\lambda / 2$, respectively. Although the array configurations for three methods are different, the degrees of array configurations are the same and the projections of the array configurations on the $x-y$ plane are L-shaped array configurations. In the typical DOA estimation, since the sources always come from above the sensor, that is, above the $x$ - $y$ plane, the azimuth angle is in the range of $0^{\circ}$ and $360^{\circ}$ and the elevation angle is in the range of $0^{\circ}$ and $90^{\circ}$. Hence, electric angles $\theta$ and $\varphi$ belong to $\left[-180^{\circ}, 180^{\circ}\right]$ and $\phi$ belongs to $\left[-180^{\circ}, 0^{\circ}\right]$. All simulation results are obtained from 100 Monte Carlo runs. In the simulations, the root mean squared error (RMSE) of 2D DOA estimation is defined as

$$
\operatorname{RMSE}=\frac{1}{K} \sum_{k=1}^{K}\left(\operatorname{RMSE}\left(\alpha_{k}\right)+\operatorname{RMSE}\left(\beta_{k}\right)\right),
$$

where $\operatorname{RMSE}\left(\alpha_{k}\right)=\left[\sum_{l=1}^{100}\left(\left(\widetilde{\alpha}_{k, l}-\alpha_{k}\right)^{2} / 100\right)\right]^{1 / 2}, \operatorname{RMSE}\left(\beta_{k}\right)=$ $\left[\sum_{l=1}^{100}\left(\left(\widetilde{\beta}_{k, l}-\beta_{k}\right)^{2} / 100\right)\right]^{1 / 2}$, and $\tilde{\alpha}_{k, l}$ and $\widetilde{\beta}_{k, l}$ are the estimates of $\alpha_{k}$ and $\beta_{k}$ in the lth run, respectively. It is well known that the grid interval serves as a tradeoff between precision and computational complexity. For all simulations, we make the coarse grid interval with $1^{\circ}$ and perform a local fine grid interval in the locations obtained by utilizing the coarse grid interval. 


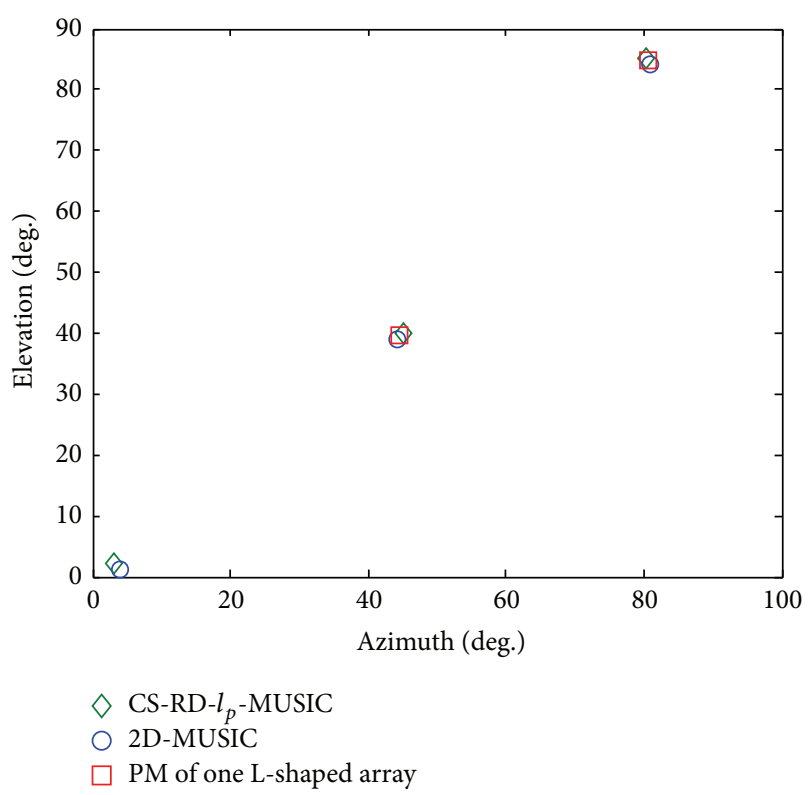

FIgURE 2: Angle estimation results of three methods for three sources.

In the first simulation, we show angle estimation results of three methods in the azimuth-elevation plane. Consider three independent sources with DOAs of $\left(\alpha_{1}=3^{\circ}, \beta_{1}=2^{\circ}\right)$, $\left(\alpha_{2}=45^{\circ}, \beta_{2}=40^{\circ}\right)$, and $\left(\alpha_{3}=80^{\circ}, \beta_{3}=85^{\circ}\right)$ impinging on the proposed array configuration. Figure 2 presents angle estimation results of three methods for all three sources with the fixed SNR $5 \mathrm{~dB}$ and number of snapshots being 100. It is indicated in Figure 2 that CS-RD- $l_{p}$-MUSIC and 2D-MUSIC can provide correct estimates for the azimuth and elevation angles of three sources but PM fails. Moreover, although CS$\mathrm{RD}-l_{p}$-MUSIC slightly outperforms $2 \mathrm{D}$-MUSIC in terms of angle estimation results, it avoids an exhaustive 2D search which is needed in 2D-MUSIC.

The RMSE of three methods versus SNR and the number of snapshots is investigated in the second simulation. We keep the same source model as in the first simulation. Figure 3 depicts RMSE as a function of SNR of three methods and CRB [23] with the fixed number of snapshots being 100, whereas RMSE versus the number of snapshots with the fixed SNR $5 \mathrm{~dB}$ is shown in Figure 4. It can be concluded from Figures 3 and 4 that CS-RD- $l_{p}$-MUSIC has more precise estimation than 2D-MUSIC and PM with no estimation failure. It is clearly seen that the performance of CS-RD- $l_{p}$-MUSIC is gradually improving and is close to the CRB with the increase of SNR and the number of snapshots.

Figure 5 illustrates the relation between the bias of DOA estimation and the angle separation of two independent sources. The bias is defined as the difference between the estimated angle and the real angle, which can indicate the degree of deviation from the true angle. The smaller the bias is, the better performance the method has. Therefore, the bias is the significant performance index. Consider two sources impinging from DOAs of $\left(\alpha_{1}=25^{\circ}, \beta_{1}=20^{\circ}\right)$ and $\left(\alpha_{2}=\right.$ $\left.\alpha_{1}+\delta, \beta_{2}=\beta_{1}+\delta\right)$, where the step of the angle separation $\delta$ is $1^{\circ}$. The SNR is $3 \mathrm{~dB}$ and the number of snapshots is 50 .

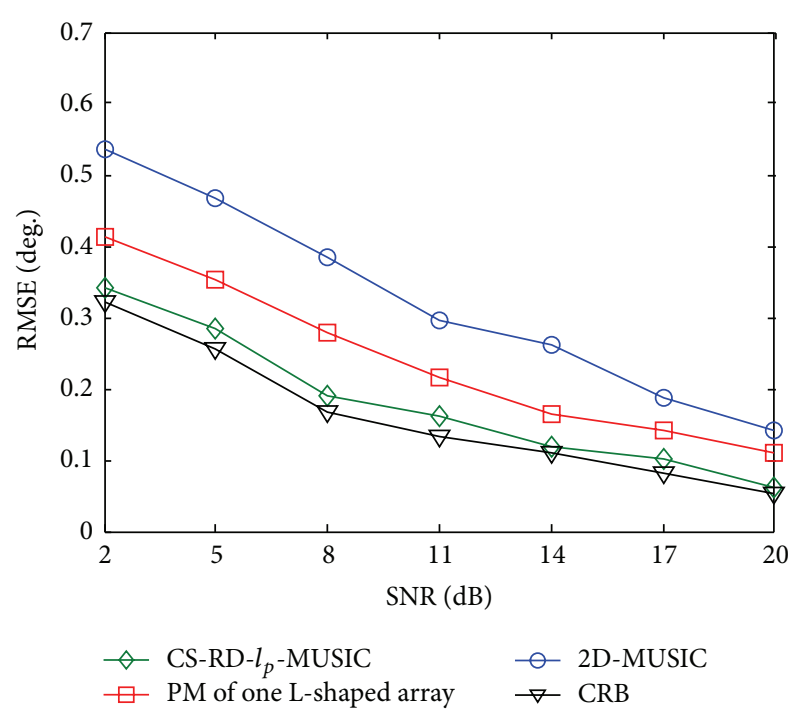

FIGURE 3: RMSE versus SNR with the fixed number of snapshots being 100 .

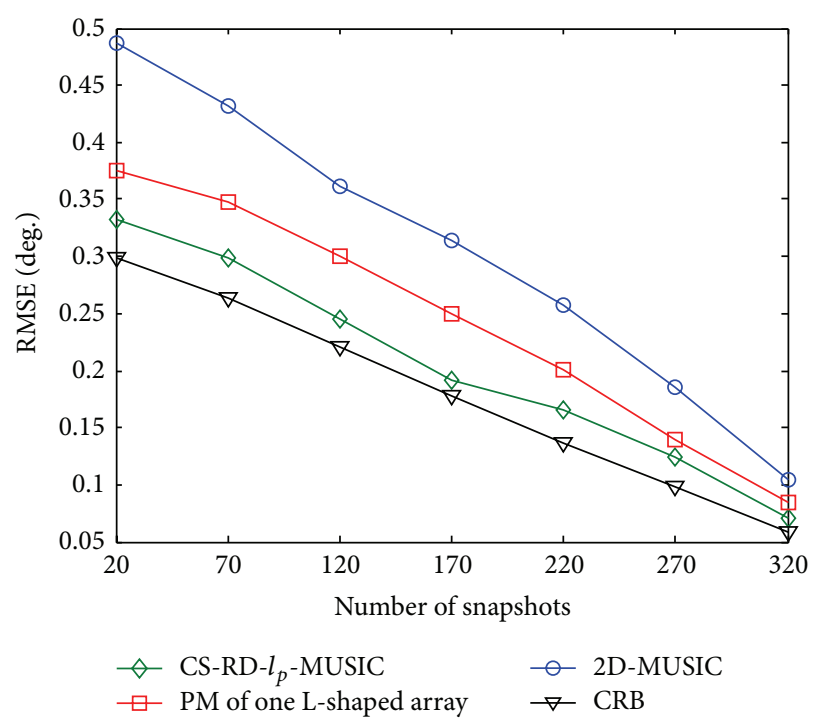

FIGURE 4: RMSE versus number of snapshots with the fixed SNR $5 \mathrm{~dB}$.

As can be seen from Figure 5, there is the bias for small angle separation using three methods and the bias of three methods disappears as long as the angle separation is no less than $17^{\circ}$.

In the fourth simulation, we compare the performance of three methods for coherent sources by showing the RMSE versus SNR and the number of snapshots. Consider two coherent sources impinging from DOAs of $\left(\alpha_{1}=30^{\circ}, \beta_{1}=\right.$ $\left.45^{\circ}\right)$ and $\left(\alpha_{2}=70^{\circ}, \beta_{2}=75^{\circ}\right)$. Since the conventional $2 \mathrm{D}$ MUSIC method is incapable of handing the coherent sources, the forward spatial smoothing method is exploited in the 2D-MUSIC called 2D-FSS-MUSIC to estimate the coherent sources. Figures 6 and 7 plot the RMSE versus SNR and the number of snapshots for coherent sources, respectively. It can be seen from Figures 6 and 7 that the proposed method has 


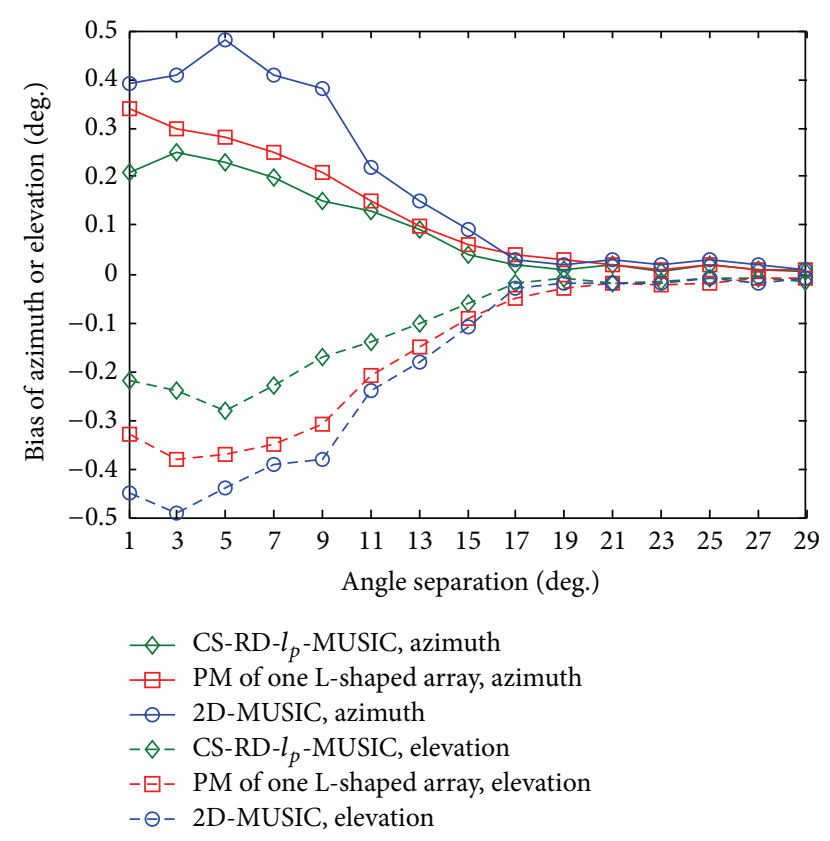

FIGURE 5: Bias versus angle separation with the fixed SNR $3 \mathrm{~dB}$ and number of snapshots being 50 .

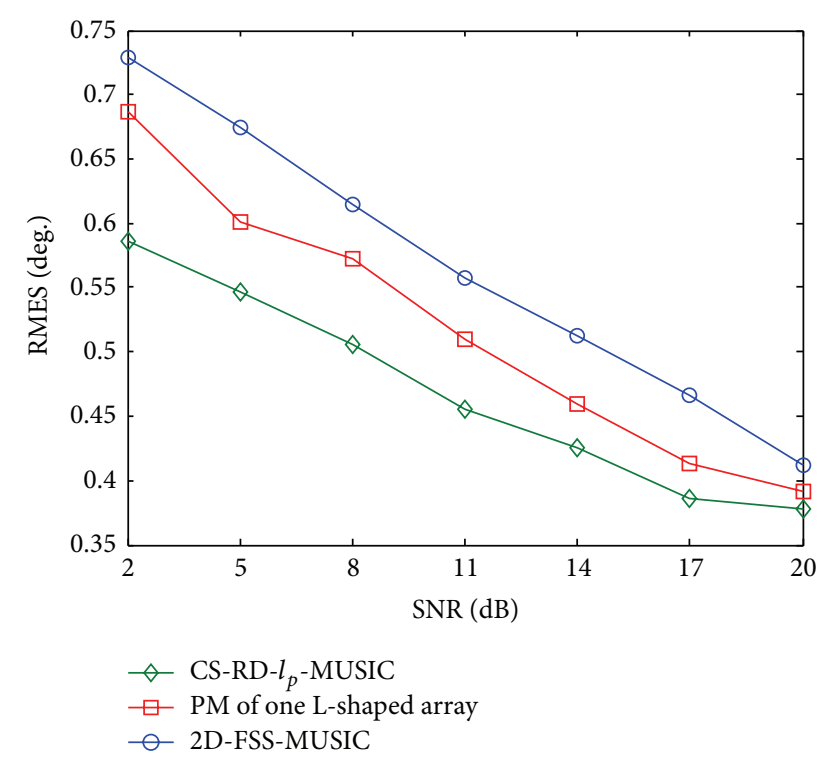

FIgURE 6: RMSE versus SNR with the fixed number of snapshots being 100 for coherent sources.

the best estimation accuracy among all three methods for coherent sources. Moreover, this performance advantage is gradually improving with SNR or the number of snapshots increasing.

Finally, Figure 8 presents the RMSE of the proposed method for all possible azimuth and elevation angles with the fixed SNR $3 \mathrm{~dB}$ and number of snapshots being 50. One single source is considered in this simulation. The steps of the azimuth and elevation angles are both fixed at $5^{\circ}$. We observe

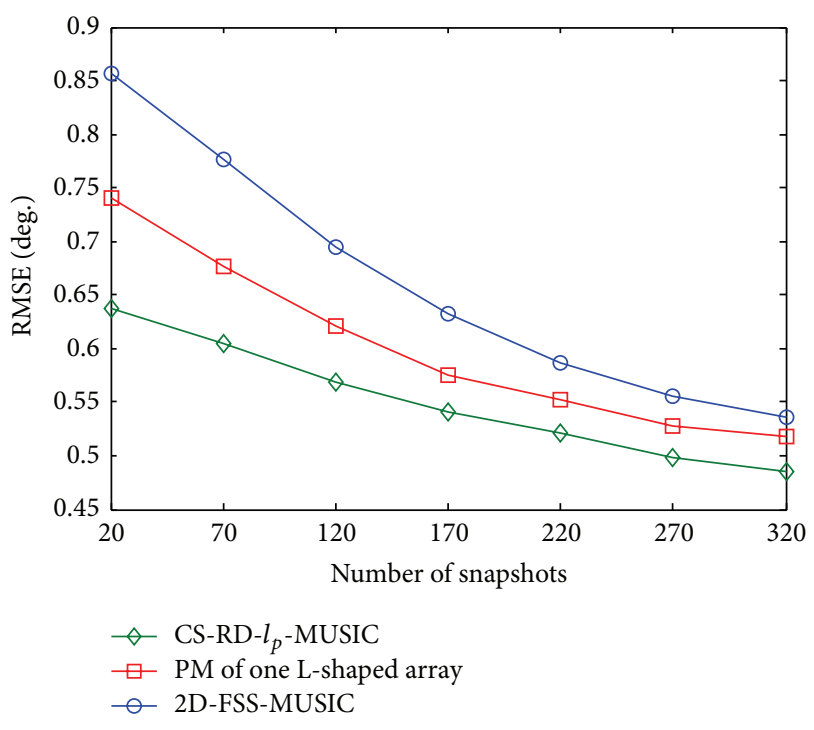

FIGURE 7: RMSE versus number of snapshots with the fixed SNR $5 \mathrm{~dB}$ for coherent sources.

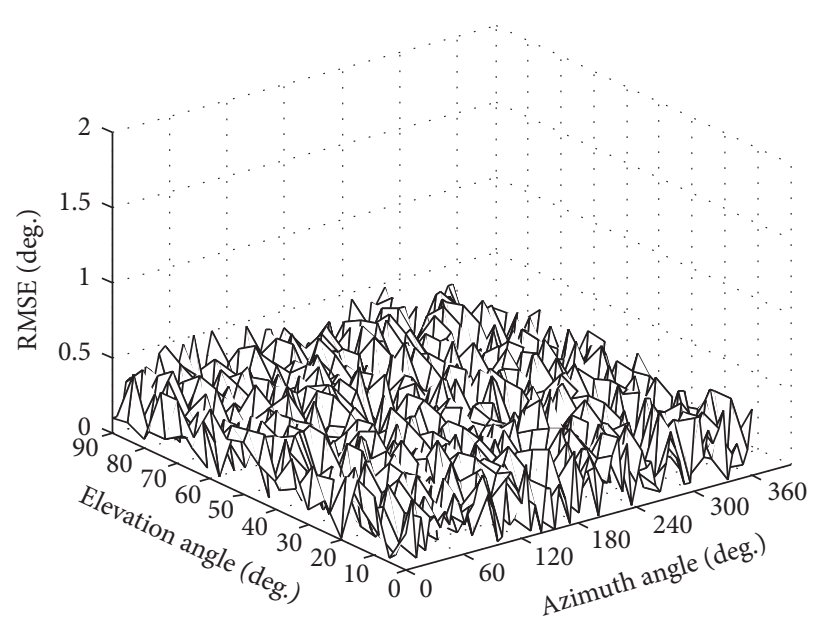

FIGURE 8: RMSE of the proposed method at different DOAs with the fixed SNR $3 \mathrm{~dB}$ and number of snapshots being 50 for one single source.

from Figure 8 that no estimation failure occurs for all pair angles with the proposed method.

\section{Conclusion}

In this paper, a novel CS-RD- $l_{p}$-MUSIC is proposed for $2 \mathrm{D}$ DOA estimation in CS. The proposed method introduces electric angles and then separates the steering vector into two parts for constructing two corresponding noise subspaces. The electric angles are estimated by CS-MUSIC and RD- $l_{p^{-}}$ MUSIC based on the constructed noise subspaces, so that the azimuth and elevation angles are obtained by arc-tangent operations in terms of estimates of electric angles. Since the arc-tangent is a one-to-one function and allows the value of its argument to be larger than unity, the proposed method never fails. The proposed method, which requires no pair 
matching, can reduce computational complexity and extend the number of snapshots to improve performance. Simulation results show that the proposed method never fails for all pair angles and has better estimation performance than PM and 2D-MUSIC in terms of RMSE and bias of DOA estimation.

\section{Conflict of Interests}

The authors declare that there is no conflict of interests regarding the publication of this paper.

\section{Acknowledgment}

This work was supported by Aviation Science Foundation of China (201401P6001).

\section{References}

[1] V. V. Reddy, M. Mubeen, and B. P. Ng, "Reduced-Complexity Super-Resolution DOA estimation with unknown number of sources," IEEE Signal Processing Letters, vol. 22, no. 6, pp. 772776, 2015

[2] Z. Tan, Y. C. Eldar, and A. Nehorai, "Direction of arrival estimation using co-prime arrays: a super resolution viewpoint," IEEE Transactions on Signal Processing, vol. 62, no. 21, pp. 55655576, 2014.

[3] R. O. Schmidt, "Multiple emitter location and signal parameter estimation," IEEE Transactions on Antennas and Propagation, vol. 34, no. 3, pp. 276-280, 1986.

[4] R. Roy and T. Kailath, "ESPRIT-estimation of signal parameters via rotational invariance techniques," IEEE Transactions on Acoustics, Speech and Signal Processing, vol. 37, no. 7, pp. 984995, 1989.

[5] D. L. Donoho, "Compressed sensing," IEEE Transactions on Information Theory, vol. 52, no. 4, pp. 1289-1306, 2006.

[6] E. J. Candès and T. Tao, "Near-optimal signal recovery from random projections: universal encoding strategies?" IEEE Transactions on Information Theory, vol. 52, no. 12, pp. 5406-5425, 2006.

[7] D. Malioutov, M. Cetin, and A. S. Willsky, "A sparse signal reconstruction perspective for source localization with sensor arrays," IEEE Transactions on Signal Processing, vol. 53, no. 8, pp. 3010-3022, 2005.

[8] P. Stoica, P. Babu, and J. Li, "SPICE: a sparse covariance-based estimation method for array processing," IEEE Transactions on Signal Processing, vol. 59, no. 2, pp. 629-638, 2011.

[9] M. M. Hyder and K. Mahata, "Direction-of-arrival estimation using a mixed $l_{2,0}$ norm approximation," IEEE Transactions on Signal Processing, vol. 58, no. 9, pp. 4646-4655, 2010.

[10] Y. Hua, T. K. Sarkar, and D. D. Weiner, "An L-shaped array for estimating 2-D directions of wave arrival," IEEE Transactions on Antennas and Propagation, vol. 39, no. 2, pp. 143-146, 1991.

[11] V. S. Kedia and B. Chandna, "A new algorithm for 2-D DOA estimation," Signal Processing, vol. 60, no. 3, pp. 325-332, 1997.

[12] M. D. Zoltowski, M. Haardt, and C. P. Mathews, "Closed-form 2-D angle estimation with rectangular arrays in element space or beamspace via unitary ESPRIT," IEEE Transactions on Signal Processing, vol. 44, no. 2, pp. 316-328, 1996.

[13] J. Ramos, C. P. Mathews, and M. D. Zoltowski, "FCA-ESPRIT: a closed-form 2-D angle estimation algorithm for filled circular arrays with arbitrary sampling lattices," IEEE Transactions on Signal Processing, vol. 47, no. 1, pp. 213-217, 1999.

[14] N. Tayem and H. M. Kwon, "L-shape 2-dimensional arrival angle estimation with propagator method," IEEE Transactions on Antennas and Propagation, vol. 53, no. 5, pp. 1622-1630, 2005.

[15] J. Liang and D. Liu, "Joint elevation and azimuth direction finding using L-shaped array," IEEE Transactions on Antennas and Propagation, vol. 58, no. 6, pp. 2136-2141, 2010.

[16] J.-F. Chen and H. Ma, "An accurate real-time algorithm for spectrum peaks search in 2D MUSIC," in Proceedings of the 2nd International Conference on Multimedia Technology, pp. 33853388, July 2011.

[17] J. F. Gu, W. P. Zhu, M. N. S. Swamy, and S. C. Chan, "Fast and accurate 2-D DOA estimation via sparse L-shaped array", in Proceedings of the IEEE International Symposium on Circuits and Systems (ISCAS '14), pp. 1292-1295, Melbourne, Australia, June 2014.

[18] F. Wang, X. Cui, and M. Lu, "Decoupled 2D direction finding based on sparse signal reconstruction," in Proceedings of the IEEE Military Communications Conference (MILCOM '14), pp. 306-311, Baltimore, Md, USA, October 2014.

[19] J. A. Tropp, "Algorithms for simultaneous sparse approximation. Part II: convex relaxation," Signal Processing, vol. 86, no. 3, pp. 589-602, 2006.

[20] S. F. Cotter, B. D. Rao, K. Engan, and K. K. Delgado, "Sparse solutions to linear inverse problems with multiple measurement vectors," IEEE Transactions on Signal Processing, vol. 53, no. 7, pp. 2477-2488, 2005.

[21] J. Chen and X. Huo, "Theoretical results on sparse representations of multiple-measurement vectors," IEEE Transactions on Signal Processing, vol. 54, no. 12, pp. 4634-4643, 2006.

[22] G. Visick, "A quantitative version of the observation that the Hadamard product is a principal submatrix of the Kronecker product," Linear Algebra and its Applications, vol. 304, no. 1-3, pp. 45-68, 2000.

[23] Y. Hua and T. K. Sarkar, "A note on the Cramer-Rao bound for 2-D direction finding based on 2-D array," IEEE Transactions on Signal Processing, vol. 39, no. 5, pp. 1215-1218, 1991. 

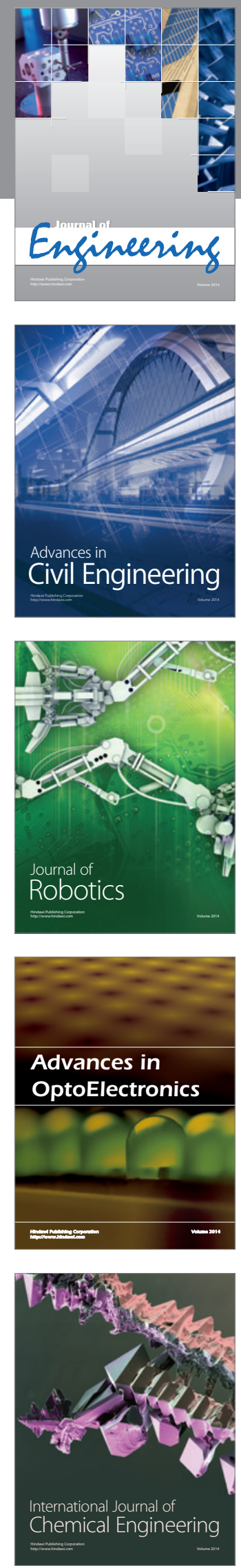

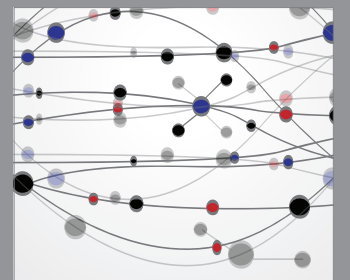

The Scientific World Journal
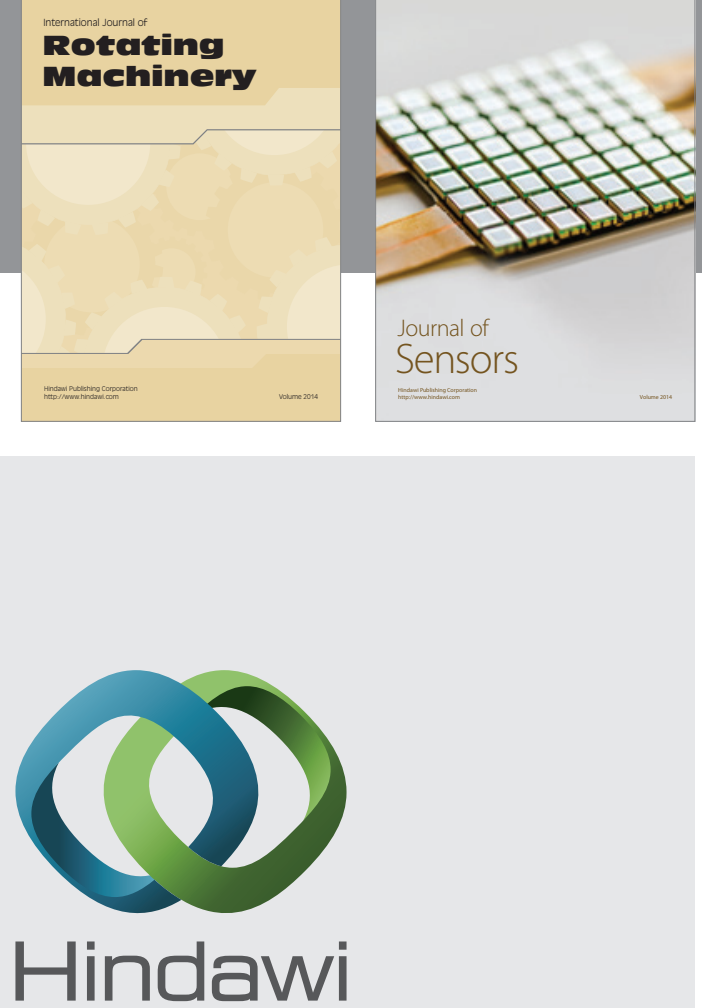

Submit your manuscripts at http://www.hindawi.com
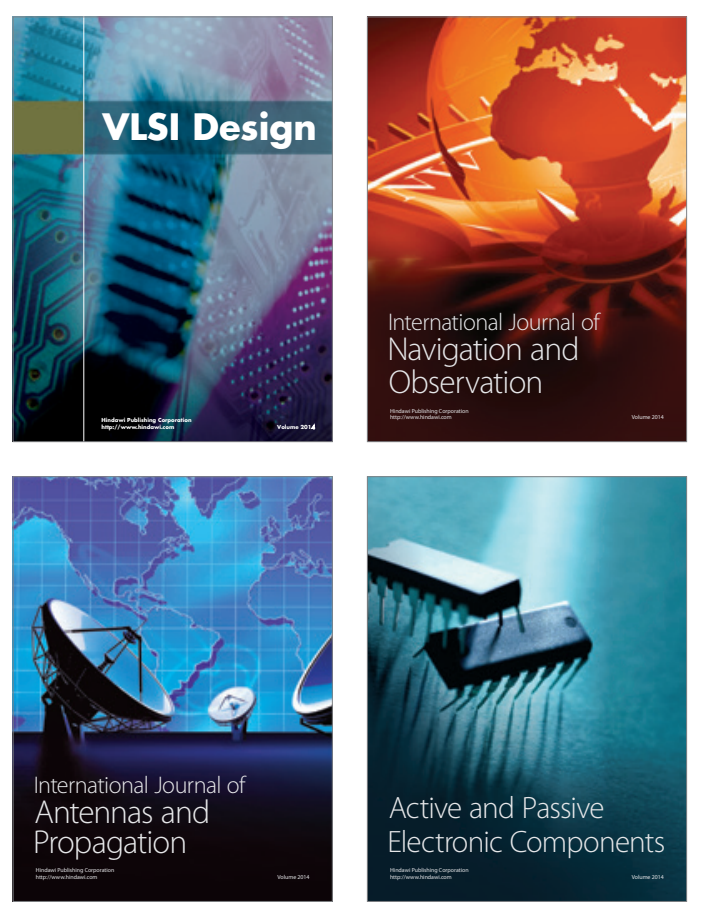
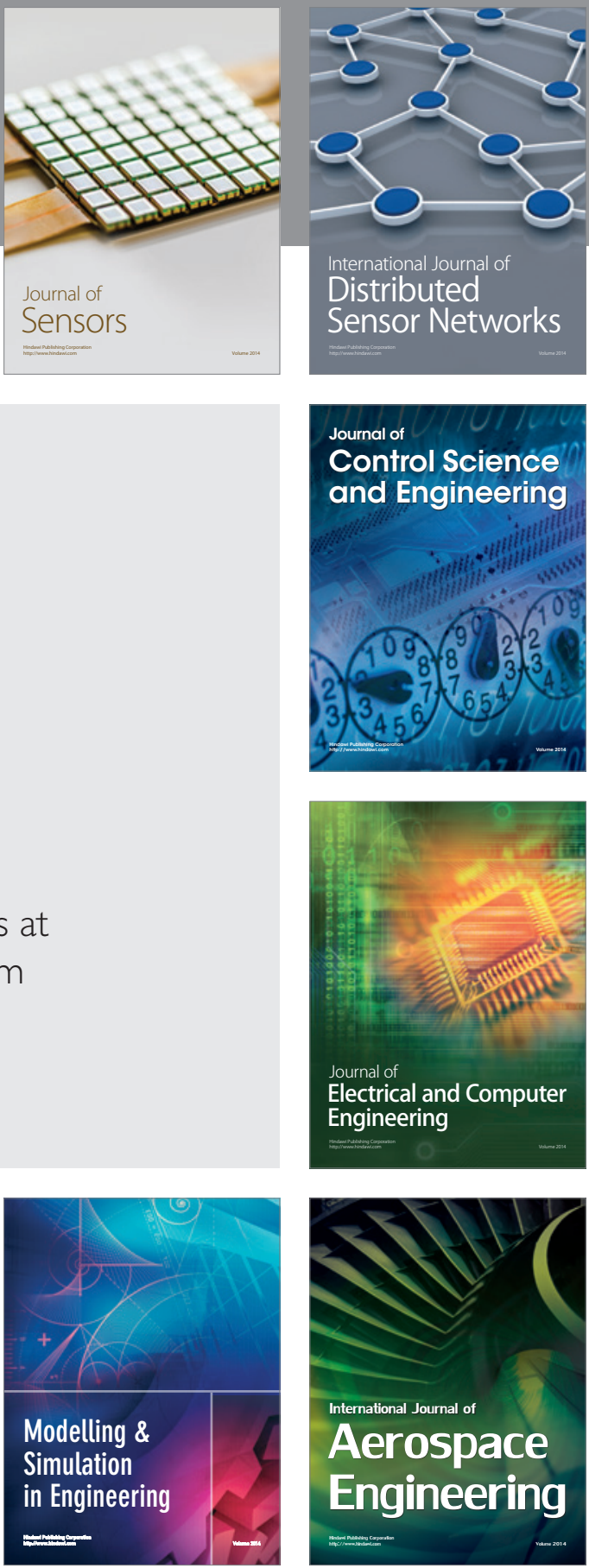

Journal of

Control Science

and Engineering
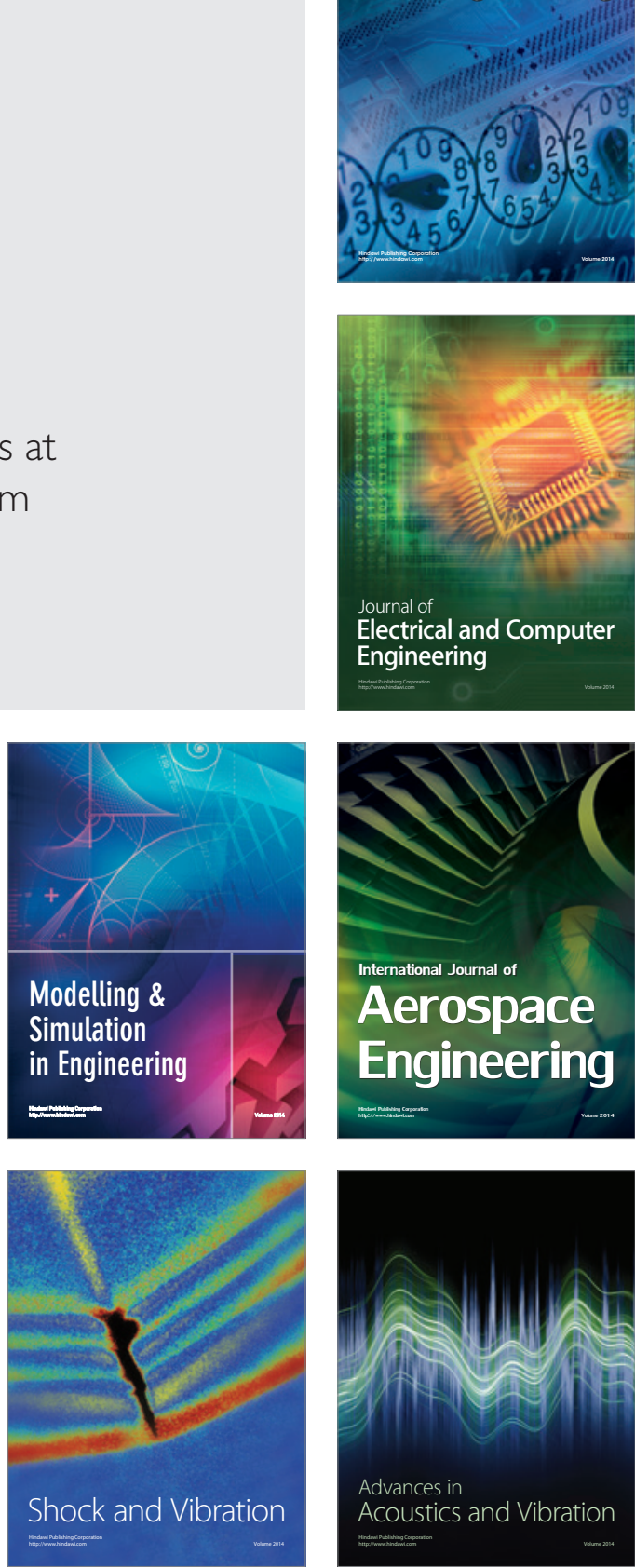